\title{
Magnetohydrodynamics in graphene: Shear and Hall viscosities
}

\author{
B. N. Narozhny ${ }^{1,2}$ and M. Schütt ${ }^{3,4,5}$ \\ ${ }^{1}$ Institut für Theorie der kondensierten Materie, Karlsruhe Institute of Technology, 76128 Karlsruhe, Germany \\ ${ }^{2}$ National Research Nuclear University MEPhI (Moscow Engineering Physics Institute), 115409 Moscow, Russia \\ ${ }^{3}$ Condensed Matter Theory Group, Paul Scherrer Institute, 5232 Villigen PSI, Switzerland \\ ${ }^{4}$ Department of Theoretical Physics, University of Geneva, 1211 Geneva, Switzerland \\ ${ }^{5}$ Institute for Theoretical Physics, ETH Zurich, 8093 Zurich, Switzerland
}

(Received 26 May 2019; published 24 July 2019)

\begin{abstract}
Viscous phenomena are the hallmark of the hydrodynamic flow exhibited by Dirac fermions in clean graphene at high enough temperatures. We report a quantitative calculation of the electronic shear and Hall viscosities in graphene based on the kinetic theory combined with the renormalization group providing a unified description at arbitrary doping levels and nonquantizing magnetic fields. At charge neutrality, the Hall viscosity vanishes, while the field-dependent shear viscosity decays from its zero-field value saturating to a nonzero value in classically strong fields. Away from charge neutrality, the field-dependent viscosity coefficients tend to agree with the semiclassical expectation.
\end{abstract}

DOI: 10.1103/PhysRevB.100.035125

Hydrodynamic behavior of charge carriers in graphene has been the focus of considerable experimental [1-14] and theoretical efforts [15-38] (recently reviewed in Refs. [39,40]). Within linear response, the difference between an Ohmic current and a hydrodynamic flow is determined by the viscosity $[4,8,14,23,25,26,30]$ (see Refs. $[9,12]$ on the issue of ballistic electrons).

In traditional hydrodynamics [41], viscosity is a measure of mutual friction between the neighboring fluid elements moving with distinct velocities. From the viewpoint of the microscopic theory, viscosity is a fourth-rank tensor that can be defined as a "response function" relating the stress (or momentum flux) to the strain [35,42]. In isotropic systems [41], the viscosity tensor contains two independent coefficients, the shear and bulk viscosities. The latter is only important for physical phenomena associated with fluid compressibility and is known to vanish for monoatomic gases [41], ultrarelativistic systems [43,44], and Fermi liquids [45]. Similarly, it's been argued to vanish in graphene $[22,25,27]$, at least to the leading approximation. In anisotropic systems the situation is more involved [46].

Treating the shear viscosity as a linear response function, one can derive a Kubo formula [27,35,42] that can be related to the Kubo formula for electrical conductivity [42]. In practice, Kubo-formula-based calculations are typically perturbative and one can only use this approach to evaluate the viscosity either in doped graphene (i.e., in the so-called "Fermi-liquid" or "degenerate" limit) [27] or in the highfrequency collisionless regime [35]. At charge neutrality, one typically turns to the kinetic theory $[16,22]$ combined with the renormalization group [47].

The relation between the Kubo formulas for shear viscosity and conductivity [42] leads to certain expectations regarding the dependence of the viscosity tensor on the external magnetic field. In the simplest case [48-50], one finds the field-dependent shear viscosity and the newly appearing Hall viscosity to mimic the magnetoconductivity and Hall conductivity in the usual Drude theory, respectively, with the only difference that the scattering time is now provided by electron-electron interactions.

An experimental measurement of the electronic shear viscosity is a nontrivial task [51]. Based on nonlocal resistance measurements [4], a related quantity — the kinematic viscosity [41] — was estimated to have a "higher-than-in-honey" value $v \approx 0.1 \mathrm{~m}^{2} / \mathrm{s}$ at typical charge densities, $n \sim 10^{12} \mathrm{~cm}^{-2}$, and temperatures, $T=220 \mathrm{~K}$. This value is of the same order of magnitude as the theoretical expectation [27] for doped graphene and agrees with more recent measurements $[8,14]$. In contrast, the theoretical result for the shear viscosity in neutral graphene $[16,22,25]$ has not been tested experimentally. Hall viscosity has been studied in a recent experiment reported in Ref. [14]. Again, the measurements were performed away from charge neutrality, where the shear and Hall viscosities follow the semiclassical field dependence $[48,49,52]$.

The purpose of the present paper is to provide a consistent, unified calculation of the shear and Hall viscosities in graphene at arbitrary doping levels within the "hydrodynamic" temperature window [39] and at arbitrary nonquantizing magnetic fields. In a companion paper [53], we have generalized the nonlinear hydrodynamic equations derived in Ref. [25] to include the external magnetic field. Here we report a quantitative calculation of the kinetic coefficients on the basis of the kinetic theory combined with the renormalization group approach. At neutrality and in the degenerate regime, the results can be obtained in a closed analytical form. For arbitrary carrier densities, the viscosities can be expressed in terms of certain "scattering rates" to be evaluated numerically.

The results of our calculations are in good agreement with available experimental evidence. In the absence of the magnetic field, we find the kinematic viscosity to depend rather weakly on the carrier density, remaining of the order of $v \approx 0.1 \mathrm{~m}^{2} / \mathrm{s}$ for all densities explored in the experiment of Ref. [4]. The field-dependent shear and Hall viscosities away from charge neutrality are qualitatively similar to the 
semiclassical expectations [14], reaching the standard Drudelike dependence at the experimental densities, $n \sim 10^{12} \mathrm{~cm}^{-2}$. In contrast, the shear viscosity at the neutrality point in classically strong magnetic fields saturates to a nonzero value. Our results are illustrated in physical units in Figs. 5 and 7.

\section{FROM THE MICROSCOPIC THEORY TO HYDRODYNAMICS}

We begin with a brief review of the hydrodynamic approach and a discussion of the applicability of hydrodynamics to Dirac fermions in graphene. The ideas presented here were developed in full detail in Refs. [15,25,44,53] (see the recent review [40] for a more detailed discussion and a complete set of references) and are included here to make the paper self-contained.

Hydrodynamics is a macroscopic manifestation of conservation laws in an interacting many-body system that is a fluid. Typically [41], one considers conservation of the particle number (or, equivalently either mass or electric charge), energy, and momentum. The latter provides the most stringent restrictions on the applicability of hydrodynamics limiting one to systems with the only (or the dominant) scattering mechanism being due to interparticle collisions (e.g., electronelectron interaction) that conserve momentum. At first glance this rules out scattering with other types of excitations (e.g., electron-phonon or electron-magnon scattering) as well as microscopically varying external potentials (e.g., potential disorder). In a typical solid, such processes dominate the linear-response transport properties and while they can be accounted for using the Boltzmann kinetic theory [44], a fully hydrodynamic description is not possible.

In recent years, a number of materials have been developed [4-6,10,11] which are, on one hand, clean enough, such that disorder scattering is important only at the lowest temperatures and on the other hand rigid enough, such that the electron-phonon interaction is relevant at much higher temperatures. This provides for a considerable intermediate temperature range $[25,39]$ where the electron-electron interaction is the dominant scattering mechanism in the system. Mathematically, the above can be summarized by the inequality,

$$
\tau_{\mathrm{ee}} \ll \tau_{\text {dis }}, \tau_{\mathrm{e} \text {-ph }}, \text { etc., }
$$

where $\tau_{\mathrm{ee}}$ is the typical time scale associated with the electron-electron interaction, $\tau_{\mathrm{dis}}$ describes disorder scattering, $\tau_{\mathrm{e}-\mathrm{ph}}$ the electron-phonon interaction, and "etc." stands for any other scattering-related time scale in the problem (e.g., the "recombination" time $\tau_{R}$, see below).

Assume that the condition (1) is sufficient to uphold the conservation laws in the electronic system. Then they can be expressed in terms of continuity equations that can be either written down on symmetry grounds [41] or can be obtained by integrating the kinetic equation [44]. The first of these equations is the standard continuity equation reflecting charge conservation

$$
\partial_{t} n+\nabla_{\boldsymbol{r}} \cdot \boldsymbol{j}=0,
$$

which in this paper we express in terms of the carrier density $n$ and current $\boldsymbol{j}$, such that the actual charge density and electric current are obtained by multiplying these quantities by the electron charge.

The second equation reflects energy conservation. In the case of charge carriers, this equation differs $[39,40]$ from its textbook counterpart $[41,44]$ by an extra term describing Joule's heat

$$
\partial_{t} n_{E}+\nabla_{\boldsymbol{r}} \cdot \boldsymbol{j}_{E}=e \boldsymbol{E} \cdot \boldsymbol{j} .
$$

Here $n_{E}$ and $\boldsymbol{j}_{E}$ are the energy density and current, $\boldsymbol{E}$ is the electric field, and $e$ is the electron charge.

The third equation describes momentum conservation. In contrast to the corresponding equation for a neutral fluid $[41,44]$, this equation takes into account the effect of electromagnetic fields. Moreover, for reasons that will become clear below, we include a small [as required by Eq. (1)] disorder-scattering term $[24,25]$

$$
\partial_{t} n_{k}^{\alpha}+\nabla_{r}^{\beta} \Pi_{E}^{\alpha \beta}-e n E^{\alpha}-\frac{e}{c}[\boldsymbol{j} \times \boldsymbol{B}]^{\alpha}=-\frac{n_{k}^{\alpha}}{\tau_{\mathrm{dis}}} .
$$

Here $\boldsymbol{n}_{\boldsymbol{k}}$ is the momentum density, $\Pi_{E}^{\alpha \beta}$ is the momentum flux (or stress tensor), and $\boldsymbol{B}$ is the magnetic field.

The continuity equations (2a)-(2c) are valid for any electronic system satisfying Eq. (1). In the particular case of graphene, one may neglect scattering processes that change the number of particles in each individual band (e.g., the Auger processes, three-particle collisions, electron-phonon interaction, etc.) such that the number of particles in each band should be conserved separately. As a result, one finds another continuity equation $[21,39,40]$

$$
\partial_{t} n_{I}+\nabla_{r} \cdot j_{I}=-\frac{n_{I}-n_{I, 0}}{\tau_{R}},
$$

where $n_{I}$ and $\boldsymbol{j}_{I}$ are the so-called "imbalance" (or total quasiparticle) density and currents that are related to the particle number densities $n_{ \pm}$and currents $\boldsymbol{j}_{ \pm}$, in the two bands as

$$
\begin{array}{ll}
n=n_{+}-n_{-}, & n_{I}=n_{+}+n_{-}, \\
\boldsymbol{j}=\boldsymbol{j}_{+}-\boldsymbol{j}_{-}, & \boldsymbol{j}_{I}=\boldsymbol{j}_{+}+\boldsymbol{j}_{-} .
\end{array}
$$

The right-hand side in Eq. (2d) describes weak [again, in the sense of Eq. (1)] quasiparticle recombination characterized by a (long) time scale $\tau_{R}$. The quantity $n_{I, 0}$ is the equilibrium density of quasiparticles.

The kinetic derivation $[25,44]$ of the continuity equations (2) has the advantage of providing "microscopic" definitions of all macroscopic quantities in Eqs. (2) in terms of the quasiparticle distribution function. In graphene (or any other twoband system), one may label the single-particle (band) states by the band index $\lambda= \pm$ and the momentum $k$. Denoting the distribution function by $f_{\lambda k}$, we define the above densities and currents as

$$
\begin{aligned}
n_{+} & =N \int \frac{d^{2} k}{(2 \pi)^{2}} f_{+, k}, \quad n_{-}=N \int \frac{d^{2} k}{(2 \pi)^{2}}\left(1-f_{-, \boldsymbol{k}}\right), \\
\boldsymbol{j} & =N \sum_{\lambda} \int \frac{d^{2} k}{(2 \pi)^{2}} \boldsymbol{v}_{\lambda k} f_{\lambda \boldsymbol{k}}, \\
\boldsymbol{j}_{I} & =N \sum_{\lambda} \int \frac{d^{2} k}{(2 \pi)^{2}} \lambda \boldsymbol{v}_{\lambda \boldsymbol{k}} f_{\lambda \boldsymbol{k}},
\end{aligned}
$$




$$
\begin{aligned}
n_{E} & =N \int \frac{d^{2} k}{(2 \pi)^{2}}\left[\epsilon_{+, k} f_{+, k}+\epsilon_{-, k}\left(f_{-, k}-1\right)\right], \\
\boldsymbol{j}_{E} & =N \sum_{\lambda} \int \frac{d^{2} k}{(2 \pi)^{2}} \epsilon_{\lambda k} \boldsymbol{v}_{\lambda \boldsymbol{k}} f_{\lambda \boldsymbol{k}}=v_{g}^{2} \boldsymbol{n}_{\boldsymbol{k}}, \\
\boldsymbol{n}_{\boldsymbol{k}} & =N \sum_{\lambda} \int \frac{d^{2} k}{(2 \pi)^{2}} \boldsymbol{k} f_{\lambda \boldsymbol{k}}, \\
\Pi_{E}^{\alpha \beta} & =N \sum_{\lambda} \int \frac{d^{2} k}{(2 \pi)^{2}} k^{\alpha} v_{\lambda \boldsymbol{k}}^{\beta} f_{\lambda \boldsymbol{k}} .
\end{aligned}
$$

Here $N=4$ is the degeneracy factor. The second equality in Eq. (3f) is specific to the Dirac spectrum in graphene and represents a crucial difference between the electron fluid in graphene and the usual (massive) fluids. Indeed, assuming the Dirac form of the quasiparticle spectrum [54]

$$
\epsilon_{\lambda k}=\lambda v_{g} k
$$

one immediately finds the following relations between velocity and momentum (where $\boldsymbol{e}$ denotes the unit vector in the direction indicated by the subscript)

$$
\boldsymbol{v}_{\lambda \boldsymbol{k}}=\lambda v_{g} \frac{\boldsymbol{k}}{k}, \quad \boldsymbol{e}_{\boldsymbol{v}}=\boldsymbol{e}_{\boldsymbol{k}}, \quad \boldsymbol{k}=\frac{\lambda k}{v_{g}} \boldsymbol{v}_{\lambda k}=\frac{\epsilon_{\lambda \boldsymbol{k}} \boldsymbol{v}_{\lambda \boldsymbol{k}}}{v_{g}^{2}} .
$$

Inserting Eqs. (5) into Eqs. (3g) and (3f), one concludes that (i) the momentum density in graphene is equivalent to the energy flux and (ii) the hydrodynamic flow in graphene describes the energy flow in contrast to the standard hydrodynamics describing the mass flow of a conventional fluid. Moreover, conservation of momentum leads to the conclusion that the energy flux in graphene is not relaxed by electronelectron interaction. The system can only reach the steady state by means of (weak) disorder scattering [24,25] described by the last term in Eq. (2c).

\section{IDEAL HYDRODYNAMICS IN GRAPHENE}

The true equilibrium state (in the absence of the external fields) is described by the Fermi-Dirac distribution function yielding constant densities and zero currents, such that each term in Eqs. (2) vanishes. Applying weak external fields one drives the system weakly out of equilibrium. This can be described either by means of the perturbative linear-response theory [24], or, if the condition (1) is fulfilled, by the hydrodynamic theory $[39,40]$. The latter approach requires two additional assumptions.

First, one assumes the spatial and temporal variations of the external fields and the resulting currents and density inhomogeneities to be small [ultimately, in the sense of Eq. (1) or the equivalent relation of the corresponding length scales], such that the electron-electron scattering processes may maintain local equilibrium. The latter is described by the distribution function $[15,25,39,40,53]$

$$
f_{\lambda \boldsymbol{k}}^{(0)}(\boldsymbol{r})=\left\{1+\exp \left[\frac{\epsilon_{\lambda \boldsymbol{k}}-\mu_{\lambda}(\boldsymbol{r})-\boldsymbol{u}(\boldsymbol{r}) \cdot \boldsymbol{k}}{T(\boldsymbol{r})}\right]\right\}^{-1},
$$

where $\mu_{\lambda}(\boldsymbol{r}), T(\boldsymbol{r})$, and $\boldsymbol{u}(\boldsymbol{r})$ are the local chemical potential, local temperature, and hydrodynamic (or "drift") velocity, respectively.
Using the distribution function (3), one finds the expressions for the equilibrium hydrodynamic quantities as well as the thermodynamic pressure $P$ and enthalpy $W$ listed in Appendix A. Substituting these quantities into the continuity Eq. (2), one obtains the (ideal) hydrodynamic equations. In particular, expressing the continuity Eq. (2c) for the momentum density in terms of the hydrodynamic velocity $\boldsymbol{u}$, we obtain the generalized Euler Eq. [41]

$$
\begin{gathered}
W\left(\partial_{t}+\boldsymbol{u} \cdot \nabla\right) \boldsymbol{u}+v_{g}^{2} \nabla P+\boldsymbol{u} \partial_{t} P+e(\boldsymbol{E} \cdot \boldsymbol{j}) \boldsymbol{u} \\
\quad=v_{g}^{2} e n \boldsymbol{E}+v_{g}^{2} \frac{e}{c} \boldsymbol{j} \times \boldsymbol{B}-\frac{W \boldsymbol{u}}{\tau_{\mathrm{dis}}} .
\end{gathered}
$$

Equation (7) was suggested in Ref. [22] in the absence of electromagnetic fields and weak disorder (terms due to the electric field were discussed in Ref. [25]). In comparison to the standard Euler equation, the generalized Eq. (7) contains two extra terms: (i) the time derivative of pressure that can be interpreted as a reminder of the relativistic nature $[15,40]$ of the quasiparticle spectrum in graphene, Eq. (4) and (ii) the disorder scattering term necessary to establish a steady state $[24,25,53]$.

Away from charge neutrality, the Euler Eq. (7) allows for a homogeneous, steady flow [24] that is equivalent to the usual Ohmic current [using Eqs. (A1)-(A5) with $\mu_{ \pm}=\mu$ and $\mu \gg T]$

$$
v_{g}^{2} e n \boldsymbol{E}+v_{g}^{2} \frac{e}{c} \boldsymbol{j} \times \boldsymbol{B}=\frac{\mu \boldsymbol{j}}{\tau_{\mathrm{dis}}},
$$

characterized by the standard Drude-like expressions for the longitudinal and Hall resistivities

$$
\rho_{x x}^{(0)}=\frac{\pi}{e^{2}|\mu| \tau_{\mathrm{dis}}}, \quad \rho_{x y}^{(0)}=R_{H} B, \quad R_{H}=-\frac{\operatorname{sgn} \mu}{n|e| c},
$$

where $R_{H}$ is the Hall coefficient.

The complete set of the equations of ideal hydrodynamics in graphene includes the generalized Euler equation (7), the continuity equations (2a), (2b), and (2d), as well as the Poisson's equation relating the charge density to the electric field and the (thermodynamic) equation of state [15,25], see Eqs. (A5),

$$
W=n_{E}+P=\frac{3 n_{E}}{2+u^{2} / v_{g}^{2}} .
$$

This equation has the thermodynamic nature and represents the second assumption needed to build the hydrodynamic theory, namely, that thermodynamic quantities and their relations are not affected by the dissipative corrections to the ideal hydrodynamics [44].

\section{GENERALIZED NAVIER-STOKES EQUATION IN GRAPHENE}

Taking into account dissipative processes modifies the macroscopic quantities and turns the ideal Euler equation into the Navier-Stokes equation, the central equation of the hydrodynamic theory [41]. Following the standard approach [39-41,44], we focus on the velocity-independent kinetic coefficients. In graphene, these include the viscosity and "quantum conductivity" (cf. the thermal conductivity in the traditional hydrodynamics [41]). 
In the usual hydrodynamics [25,40,41,53], viscosity is defined as the coefficient in the leading term of the gradient expansion of the dissipative correction to the stress tensor

$$
\begin{aligned}
\Pi_{E}^{\alpha \beta} & =\Pi_{E, 0}^{\alpha \beta}+\delta \Pi_{E}^{\alpha \beta}, \\
\delta \Pi_{E}^{\alpha \beta} & =-\eta(B) \mathfrak{D}^{\alpha \beta}+\eta_{H}(B) \epsilon^{\alpha i j} \mathfrak{D}^{i \beta} e_{B}^{j}, \\
\mathfrak{D}^{\alpha \beta} & =\nabla^{\alpha} u^{\beta}+\nabla^{\beta} u^{\alpha}-\delta^{\alpha \beta} \nabla \cdot \boldsymbol{u},
\end{aligned}
$$

where $\boldsymbol{e}_{B}=\boldsymbol{B} / B, \eta(B)$, and $\eta_{H}(B)$ are the field dependent shear [48-50] and Hall [42,48-50,52,53] viscosities (the latter appears only in the presence of magnetic field; bulk viscosity in graphene vanishes, at least to the leading approximation $[25,39,40])$. While the sign of $\eta$ is fixed by thermodynamics [41,44], the sign of $\eta_{H}$ is not. Similarly to Ref. [14], we adopt the convention where the Hall viscosity is positive for electrons [53] (and negative for holes).

Substituting Eqs. (9) into the continuity equation (2c) and repeating the steps leading to the Euler Eq. (7), we obtain the generalized Navier-Stokes equation [53]

$$
\begin{aligned}
& W\left(\partial_{t}+\boldsymbol{u} \cdot \nabla\right) \boldsymbol{u}+v_{g}^{2} \nabla P+\boldsymbol{u} \partial_{t} P+e(\boldsymbol{E} \cdot \boldsymbol{j}) \boldsymbol{u} \\
& \quad=v_{g}^{2}\left[\eta \Delta \boldsymbol{u}-\eta_{H} \Delta \boldsymbol{u} \times \boldsymbol{e}_{B}+e n \boldsymbol{E}+\frac{e}{c} \boldsymbol{j} \times \boldsymbol{B}\right]-\frac{\boldsymbol{j}_{E}}{\tau_{\mathrm{dis}}} .
\end{aligned}
$$

Comparing the first terms in the left- and right-hand sides of Eq. (10), we define the kinematic viscosity

$$
v=v_{g}^{2} \eta / W
$$

with the dimensionality of the diffusion constant $\left(\mathrm{m}^{2} / \mathrm{s}\right)$.

The second set of the kinetic coefficients describes the dissipative corrections to the quasiparticle currents $[25,53]$ (in the presence of disorder, the energy current acquires a dissipative correction of its own):

$$
\begin{aligned}
\boldsymbol{j}= & n \boldsymbol{u}+\delta \boldsymbol{j}, \quad \boldsymbol{j}_{I}=n_{I} \boldsymbol{u}+\delta \dot{j}_{I}, \quad j_{E}=W \boldsymbol{u} \\
T & \widehat{\widehat{\Sigma}}\left(\begin{array}{c}
e \boldsymbol{E}+\frac{e}{c} \boldsymbol{u} \times \boldsymbol{B}-T \nabla \frac{\mu}{T} \\
T \nabla \frac{\mu_{I}}{T} \\
0
\end{array}\right) \\
& +\widehat{\Sigma}_{H}\left(\begin{array}{c}
e \boldsymbol{E}+\frac{e}{c} \boldsymbol{u} \times \boldsymbol{B}-T \nabla \frac{\mu}{T} \\
T \nabla \frac{\mu_{I}}{T} \\
0
\end{array}\right) \times \boldsymbol{e}_{B}, \\
\mu= & \left(\mu_{+}+\mu_{-}\right) / 2, \quad \mu_{I}=\left(\mu_{+}-\mu_{-}\right) / 2 .
\end{aligned}
$$

The "imbalance" chemical potential $\mu_{I}$ is relevant for thermoelectric effects [21], which will be considered elsewhere. In this paper we disregard the possibility of the temperature gradients and set $\mu_{ \pm}=\mu$ (or $\mu_{I}=0$ ).

At charge neutrality, the matrix $\widehat{\Sigma}$ is block diagonal, i.e., the electric current decouples from the energy and imbalance currents. For $n=0$, the total current $\boldsymbol{j}$ is given by the dissipative correction $\delta \boldsymbol{j}$ which remains finite even in the absence of disorder, $\tau_{\text {dis }} \rightarrow \infty$,

$$
e \delta \boldsymbol{j}(\mu=0)=\sigma_{Q} \boldsymbol{E}, \quad \sigma_{Q}=\mathcal{A} e^{2} / \alpha_{g}^{2}, \quad \mathcal{A} \approx 0.12 .
$$

Here $\sigma_{Q}$ is known as the "quantum" or "intrinsic" conductivity of graphene $[16,17,25,40,53,55]$. Within the electronic hydrodynamics in graphene, this quantity appears instead of the usual thermal conductivity due to the special relation between the energy current and momentum, see Eq. (3f) and the discussion following Eq. (5).

Together with the continuity equations (2) and the equation of state (8), the generalized Navier-Stokes equation (10) forms a closed system of hydrodynamic equations that can be solved in arbitrary geometries (see Ref. [36] for the appropriate boundary conditions). Away from charge neutrality, these equations have to be solved together with the electrostatics equations similarly to the usual Vlasov self-consistency $[25,44]$. In free (e.g., suspended) graphene the latter is given by the Poisson's equation. In gated structures used in the majority of experiments $[4,8,9,14]$ the electrostatics is dictated by the gate $[56,57]$, simplifying the relation between the charge density and the electric field.

\section{KINETIC CALCULATION OF ELECTRONIC VISCOSITY IN GRAPHENE}

In this section we report the kinetic theory results for the electronic viscosity in graphene. The calculation method was outlined in Ref. [25] but unfortunately involves some tedious algebra yielding the viscosity coefficients in terms of rather cumbersome multidimensional integrals, see Appendix B. In the simple limiting cases (e.g., at charge neutrality and in the degenerate, "Fermi-liquid" regime), analytical results can be obtained. Otherwise, for arbitrary doping levels the shear and Hall viscosities, see Eqs. (B1), are computed numerically. These results are illustrated in Figs. 1-3. Details of the derivation are published in Ref. [53].

The kinetic theory is formally valid only in the weak coupling limit. In particular, the "collinear" (or "three-mode") approximation $[18,25,53]$ that allows us to solve the kinetic equation is formally justified in the limit $\left|\ln \alpha_{g}\right| \gg 1\left(\alpha_{g}\right.$ is the coupling constant in graphene). Therefore, in order to obtain experimentally relevant numerical values for the viscosity coefficients we supplement the kinetic theory calculation with the renormalization group analysis described in the following section.

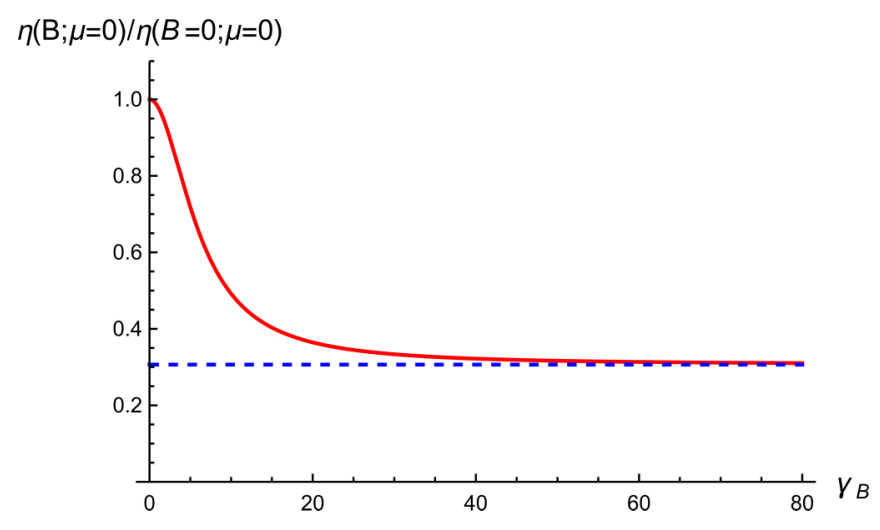

FIG. 1. Shear viscosity in graphene at charge neutrality. The solid red curve shows the ratio of the field-dependent viscosity to its zero-field value, $\eta(B ; \mu=0) / \eta(B=0 ; \mu=0)$, as a function of $\gamma_{B}$, see Eqs. (14) and (15). In classically strong fields the viscosity saturates to $\eta / \eta(B=0)=\mathcal{B}_{1} /\left(\mathcal{B B}_{2}\right) \approx 0.3065$ (for unscreened interaction) shown by the blue dashed line. 


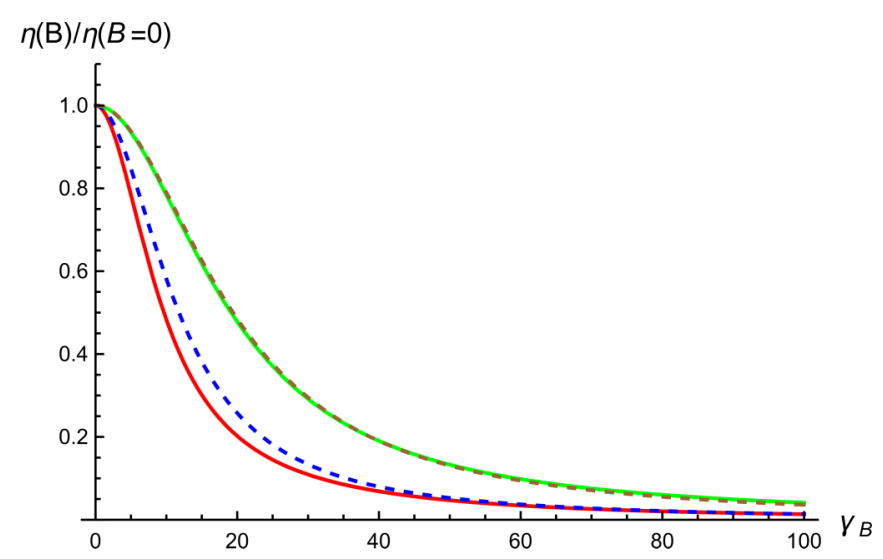

FIG. 2. Field-dependent shear viscosity in graphene away from charge neutrality. Solid curves are the result of the direct numerical evaluation of Eq. (B1a) with $\widetilde{U}=1$. Dashed curves show the "Fermiliquid" asymptotics (18). The lower (red) solid curve corresponds to $\mu / T=3$, the upper (green)- to $\mu / T=6$.

\section{A. Shear viscosity at charge neutrality}

At charge neutrality, the general expressions (B1) simplify and can be evaluated analytically [22,25] (up to a multiplicative numerical factor). The Hall viscosity vanishes identically (due to the exact electron-hole symmetry), while the shear viscosity exhibits the following behavior.

In zero magnetic field, we recover the well-known result [22] (the parametric dependence follows from the fact that the only energy scale in neutral graphene is $T$ )

$$
\eta(B=0 ; \mu=0)=\mathcal{B} \frac{T^{2}}{\alpha_{g}^{2} v_{g}^{2}} .
$$

The numerical coefficient was first evaluated in Ref. [22] reporting the value $\mathcal{B}=0.45$. Although not explicitly discussed [22], this result was obtained using the "bare" Coulomb interaction, i.e., neglecting screening effects. Such an approach is formally valid for asymptotically low temperatures $[16,18,55]$ where the coupling constant $\alpha_{g}$ is expected to have been renormalized to a small enough value (see below

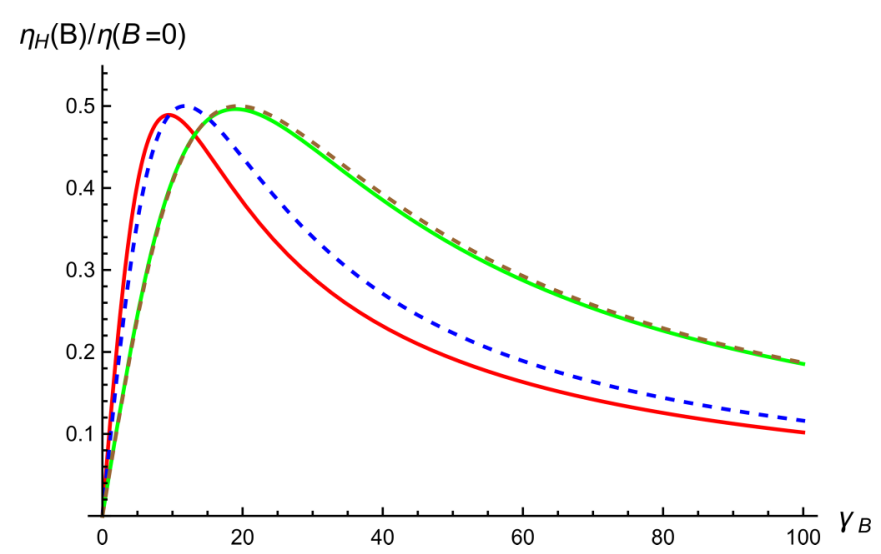

FIG. 3. Hall viscosity in graphene as a function of the magnetic field. Solid curves are the result of the direct numerical evaluation of Eq. (B1b). Dashed curves show the "Fermi-liquid" asymptotics (18). The lower (red) solid curve corresponds to $\mu / T=3$, the upper (green)-to $\mu / T=6$. for the discussion of the renormalization group approach). Indeed, evaluating the integrals (B3) for the "scattering rates" with unscreened Coulomb interaction numerically, we find $\mathcal{B}=0.446 \pm 0.005$, where the deviation stems from the systematic differences between various numerical methods. The small difference between the above result and that of Ref. [22] is due to the fact that our calculation takes into account only the direct interaction, while the exchange term is small $[16,55]$ in $1 / N$.

In magnetic field, the shear viscosity decreases but remains finite in classically strong fields (due to the aforementioned decoupling of the charge mode)

$$
\eta(B ; \mu=0)=\frac{T^{2}}{\alpha_{g}^{2} v_{g}^{2}} \frac{\mathcal{B}+\mathcal{B}_{1} \gamma_{B}^{2}}{1+\mathcal{B}_{2} \gamma_{B}^{2}}
$$

where

$$
\gamma_{B}=\frac{|e| v_{g}^{2} B}{\alpha_{g}^{2} c T^{2}}
$$

and, again neglecting screening effects, $\mathcal{B}_{1} \approx 0.0037$ and $\mathcal{B}_{2} \approx 0.0274$. The field dependence of the shear viscosity at charge neutrality is illustrated in Fig. 1.

\section{B. Viscosity away from charge neutrality}

Away from charge neutrality, the shear viscosity (B1a) has to be evaluated numerically, with the exception of the so-called "Fermi-liquid" or degenerate regime, $\mu \gg T$. In that limit, the momentum integral in Eq. (B3) is dominated by the momenta $Q>W$ [for definition of the dimensionless variables see Eq. (B7)] and can be evaluated analytically. For example (here $x=\mu / T)$,

$$
\frac{1}{\tilde{\tau}_{33}}=\frac{16 N}{\pi} \alpha_{g}^{2} \mu \int_{0}^{\infty} \frac{W^{2} d W}{\sinh ^{2} W} J_{33}\left(\alpha_{g}, W, x\right),
$$

where (here $\widetilde{Q}=Q / x, \widetilde{W}=W / x)$

$$
J_{33}=\int_{\widetilde{W}}^{1} \frac{d \widetilde{Q}}{\widetilde{Q}}|\widetilde{U}|^{2}\left(1-\frac{\widetilde{W}^{2}}{\widetilde{Q}^{2}}\right)\left(1-\widetilde{Q}^{2}\right)^{2}\left[1-\frac{\widetilde{W}^{2}}{\widetilde{Q}^{2}}\left(1-\widetilde{Q}^{2}\right)\right] \text {. }
$$

Unscreened Coulomb interaction corresponds to $\widetilde{U}=1$ (see below for a discussion of the screening effects).

Evaluating the momentum integral $J_{33}$ for unscreened Coulomb interaction in the limit $\mu \gg T$ yields

$$
J_{33}\left(\alpha_{g}=0, x \gg 1\right) \approx \ln \frac{x}{W}-\frac{3}{2},
$$

which together with the rest of the integrals (B3) leads to the following expression for the shear viscosity

$$
\eta(\mu \gg T)=\frac{3 \mu^{4}}{128 \pi^{2} \alpha_{g}^{2} v_{g}^{2} T^{2}} \frac{1}{\ln \frac{\mu}{T}+\delta_{1}-\frac{7}{4}},
$$

where $\delta_{1} \approx 0.34$. The numerical factors in the denominator represent small corrections to the leading behavior that have to be taken into account since otherwise the matrix of the scattering rates (B2f) is degenerate.

Once the magnetic field is applied, the shear viscosity (B1a) decreases, while the Hall viscosity (B1b) becomes 
nonzero. In classically strong fields both viscosities vanish (in contrast to the behavior at $\mu=0$ ). In the degenerate regime, the field dependence of the viscosity coefficients follows the simple semiclassical expectation $[14,48,49]$ (similarly to the Drude conductivity tensor):

$$
\begin{aligned}
\eta(B ; \mu \gg T) & =\frac{\eta(B=0 ; \mu \gg T)}{1+\Gamma_{B}^{2}}, \\
\eta_{H}(B ; \mu \gg T) & =\eta(B=0 ; \mu \gg T) \frac{\Gamma_{B}}{1+\Gamma_{B}^{2}},
\end{aligned}
$$

where

$$
\Gamma_{B}=2 \omega_{B} \tilde{\tau}_{11}, \quad \omega_{B}=|e| v_{g}^{2} B /(\mu c) .
$$

The field dependence (18) was suggested in Refs. [48,50] for a single-component Fermi liquid. In graphene in the degenerate limit, essentially only the single band contributes to physical observables and hence one expects to recover the single-band results. The kinetic calculation allows us to give a precise definition to the scattering rate $\tilde{\tau}_{11}$ appearing in Eqs. (18). Indeed, this rate differs [25,27] from the transport scattering rate $[55,58]$, determining the electrical conductivity as well as from the "quantum" scattering rate [55] determining the quasiparticle lifetime.

In Figs. 2 and 3 we compare the results of the numerical evaluation (with unscreened Coulomb interaction, for simplicity) of the shear viscosity (B1a) and Hall viscosity (B1b) with the asymptotic expressions (18). Qualitatively, the shape of the field dependence is the same for all values of the chemical potential. The semiclassical dependence (18) becomes indistinguishable from the full result at $\mu / T \approx 5$.

\section{RENORMALIZATION GROUP APPROACH}

Quantitative evaluation of the shear and Hall viscosities (B1a) and (B1b) in physical units requires the knowledge of the coupling constant $\alpha_{g}$. Following Refs. [22,46,47] we treat this constant as a running coupling constant in the sense of the renormalization group (RG). The final values for physical observables are then obtained by combining the RG with the scaling laws for these quantities.

The one-loop Kadanoff-Wilson RG approach to interacting Dirac fermions in graphene was suggested in Refs. [47,59,60]. The idea is to relate physical observables to their counterparts at the specifically chosen renormalization scale, where the renormalized theory is characterized by weak coupling and the kinetic theory is justified.

The renormalized carrier density obeys the relation [47]

$$
n\left(T, \mu, \alpha_{g}\right)=b^{-2} n\left[Z_{T}^{-1}(b) T, Z_{T}^{-1}(b) \mu, \alpha_{g}(b)\right] .
$$

Here $T(b)=Z_{T}^{-1}(b) T$ and $\alpha_{g}(b)=b^{-1} Z_{T}^{-1}(b) \alpha_{g}$ are the solutions of the RG equations. The latter are only valid in the lowtemperature quantum limit, $T(b)<T_{*}$, where $T_{*}$ is related to the bandwidth. Choosing the renormalization condition for $T=0$ and $\mu>0$, one finds $\mu\left(b_{*}\right)=T_{*}$, leading to the zero-temperature carrier density [47]

$$
n(T=0 ; \mu)=\frac{N \mu^{2}}{4 \pi v_{g}^{2}}\left(1+\frac{\alpha_{g}}{4} \ln \frac{T_{*}}{\mu}\right)^{-2} .
$$

For the nondegenerate system, $\mu \ll T$, the renormalization condition is essentially the same as at criticality [61] (e.g., neutral graphene), $T\left(b_{*}\right)=T_{*}$, leading to

$$
b_{*}=\frac{T_{*}}{T}\left(1+\frac{\alpha_{g}}{4} \ln \frac{T_{*}}{T}\right), \quad Z_{T}\left(b_{*}\right)=\frac{1}{b_{*}}\left(1+\frac{\alpha_{g}}{4} \ln \frac{T_{*}}{T}\right) .
$$

For general $\mu$ and $T$, the leading behavior is captured by

$$
n(T, \mu)=\frac{N T^{2}}{2 \pi v_{g}^{2}} \frac{\tilde{n}(x)}{R_{\Lambda}^{2}}, \quad R_{\Lambda}=1+\frac{\alpha_{g}}{4} \ln \frac{T_{*}}{\max (\mu, T)},
$$

where $\tilde{n}(x)$ is a dimensionless function of ratio $x=\mu / T$ that can be read off Eq. (A1a) (for $\boldsymbol{u}=0$ ). The ratio $x=\mu / T$ does not change under the RG, hence only the explicit dependence on the velocity $v_{g}$ is renormalized [16]. At the same time, the renormalized coupling constant is

$$
\alpha_{g}=\alpha_{g}^{(0)} / R_{\Lambda}
$$

The "bare" coupling constant in suspended graphene is $e^{2} /\left(\hbar v_{g}\right) \approx 2.2$ (corresponding to the "bare" velocity $v_{g} \approx 10^{6} \mathrm{~m} / \mathrm{s}$ ), while for graphene encapsulated in boron nitride [4] this reduces to $e^{2} /\left(\hbar \varepsilon v_{g}\right) \approx 0.5$ (here $\varepsilon$ is the effective dielectric constant). Assuming the value $T_{*} \approx 8.34 \times 10^{4} \mathrm{~K}$, see Refs. [47,62], and $\alpha_{g}^{(0)} \approx 0.5$ we estimate the effective coupling constant in neutral graphene

$$
\alpha_{g}(T \approx 200 \mathrm{~K}) \approx 0.285, \quad \alpha_{g}(T \approx 10 \mathrm{mK}) \approx 0.167 .
$$

Even assuming a smaller, "Fermi-liquid" coupling constant, $\alpha_{g}^{(0)} \approx 0.2$, derived from earlier measurements [63,64], we find the renormalized value of 0.11 at the lowest temperatures and 0.15 in the hydrodynamic range.

For realistic values of the carrier density in graphene in the degenerate regime $[4,8,9,14]$, the logarithmic renormalization (20) is appreciable, see Fig. 4. For $T=300 \mathrm{~K}$, the dimensionful prefactor in Eq. (20) has the value

$$
\left.\frac{N T^{2}}{2 \pi v_{g}^{2}} \rightarrow \frac{2 k_{B}^{2} T^{2}}{\pi \hbar^{2} v_{g}^{2}}\right|_{T=300 \mathrm{~K}} \approx 0.098 \times 10^{12} \mathrm{~cm}^{-2} .
$$

Neglecting the renormalization factor in Eq. (20) and using the "Fermi-liquid" asymptotics, $\tilde{n}=x^{2} / 2+\pi^{2} / 3$, we estimate the chemical potential corresponding to the typical density,

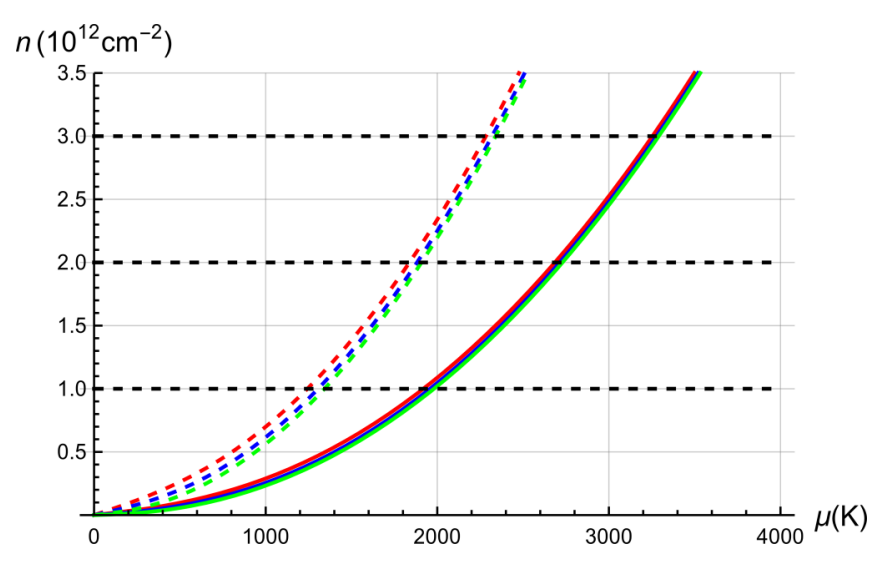

FIG. 4. Renormalized carrier density (20). The red, blue, and green solid curves correspond to $T=300,200,100 \mathrm{~K}$, respectively. Dashed curves show the nonrenormalized density. 
$n=10^{12} \mathrm{~cm}^{-2}$, as

$\mu=\sqrt{\pi k_{B}^{-2} \hbar^{2} v_{g}^{2} n\left(1-\frac{\pi k_{B}^{2} T^{2}}{3 \hbar^{2} v_{g}^{2} n}\right)} \approx 1239.7 \mathrm{~K}, \quad x \approx 4.13$.

Restoring the renormalization factor, we find increased values for the chemical potential, see Fig. 4 (the values shown in the figure were evaluated for $\alpha_{g}^{(0)} \approx 0.5$ ). For the same carrier density we find

$\mu\left(n=10^{12} \mathrm{~cm}^{-2}\right) \approx 1918.8 \mathrm{~K}, \quad x \approx 6.396, \quad R_{\Lambda} \approx 1.47$,

with the corresponding renormalized coupling constant

$$
\alpha_{g} \approx 0.34 \text {. }
$$

For the smaller "bare" coupling constant, $\alpha_{g}^{(0)} \approx 0.2$, the above values change to

$\mu\left(n=10^{12} \mathrm{~cm}^{-2}\right) \approx 1533.1 \mathrm{~K}, \quad \alpha_{g} \approx 0.167, \quad R_{\Lambda} \approx 1.2$.

Neither renormalization factor is negligible leading to a strong enhancement of the results of the kinetic theory.

Similarly to Eq. (19), the viscosity renormalizes as $[22,46]$

$$
\eta\left(T, \mu, \alpha_{g}, B\right)=\frac{1}{b^{2}} \eta\left[Z_{T}^{-1}(b) T, Z_{T}^{-1}(b) \mu, \alpha_{g}(b), b^{2} B\right],
$$

where $B$ is the magnetic field. Representing the weakcoupling kinetic-theory result (B1a) as

$$
\eta=\frac{T^{2}}{v_{g}^{2} \alpha_{g}^{2}} \tilde{\eta}\left(x, \Gamma_{B}^{i j}\right),
$$

one finds $[16,22]$ that the product $v_{g} \alpha_{g}$ doesn't renormalize. This leads to the conclusion [47] that the kinetic expression for the shear viscosity at charge neutrality, Eq. (14), provides the correct low temperature result for interacting Dirac fermions in graphene (for $B=0$ ). Implicit to this argument is the assumption of the large screening length [16]. Indeed, static screening is determined by the real part of the polarization operator, $\Pi^{R}(\omega=0)$, which for small enough momenta is well approximated by the density of states [58],

$$
\varkappa=2 \pi \alpha_{g} v_{g} \Pi^{R}(\omega=0) \approx 4 \alpha_{g} \frac{\mathcal{T}}{v_{g} R_{\Lambda}^{2}},
$$

where $\mathcal{T}=2 T \ln [2 \cosh (\mu / 2 T)]$, see Eq. (B2b). The scaling of the density of states was derived in Refs. [47,65]. The quantities $\Gamma_{B}^{i j}$ are defined similarly to Eq. (18c)

$$
\Gamma_{B}^{i j}=2 \omega_{B} \tilde{\tau}_{i j}=\frac{|e| v_{g}^{2} B}{c \alpha_{g}^{2} T^{2}} f_{i j}(x, \varkappa) R_{\Lambda}^{4},
$$

where the dimensionless functions $f_{i j}(x, \varkappa)$ can be read off Eqs. (18c) and (B3).

Finally, the kinematic viscosity (11) is a combination of the shear viscosity, renormalized velocity, and energy density. The latter renormalizes as the free energy [47]

$$
n_{E}(T, \mu, \alpha)=\frac{Z_{T}(b)}{b^{2}} n_{E}\left[Z_{T}^{-1}(b) T, Z_{T}^{-1}(b) \mu, \alpha_{g}(b)\right],
$$

which yields

$$
n_{E}(T, \mu, \alpha)=\frac{N T^{3}}{\pi v_{g}^{2}} \frac{\tilde{n}_{E}(x)}{R_{\Lambda}^{2}} .
$$

The kinematic viscosity (11) can be obtained by combining Eqs. (23) and (26) with the equation of state (8). The result is given by

$$
\nu(T, \mu, \alpha)=\frac{2 \pi v_{g}^{2} \tilde{\eta}\left(x, \Gamma_{B}^{i j}\right)}{3 N \alpha_{g}^{2} T \tilde{n}_{E}(x)} R_{\Lambda}^{4} .
$$

The results of the one-loop RG approach reviewed in this section should be treated with care. Ultimately, this is a perturbative calculation that is formally valid for weak coupling. Strictly speaking, this is not the case in real graphene (see the above estimates for the effective coupling constant) and hence the kinetic coefficients, such as viscosity, should be considered phenomenologically and assigned the experimentally measured values [40]. Nevertheless, it is instructive to evaluate the expressions for kinetic coefficients with the corresponding renormalizations to obtain a quantitative theoretical expectation for physical observables.

\section{QUANTITATIVE RESULTS FOR ELECTRONIC VISCOSITY IN GRAPHENE}

In this section we report the results of the numerical evaluation of the shear and Hall viscosity in graphene (B1) taking into account the renormalization and screening effects.

\section{A. Screening effects}

The above analytical results were obtained for the bare Coulomb interaction and are valid only in the limit of the infinitesimal interaction strength, $\alpha_{g} \rightarrow 0$. For more realistic values of $\alpha_{g}$, screening effects have to be taken into account. Within the RPA approximation $[40,55,58]$ the dynamically screened Coulomb interaction is given by

$$
\mathcal{D}_{R P A}^{R}(\omega, \boldsymbol{q})=\frac{U_{0}}{1+U_{0} \Pi^{R}(\omega, \boldsymbol{q})}, \quad U_{0}=\frac{2 \pi e^{2}}{q},
$$

where $\Pi^{R}(\omega, \boldsymbol{q})$ is the polarization operator (for a detailed calculation of the polarization operator see, e.g., Ref. [58]). In the dimensionless form of Eq. (16) one finds

$$
\widetilde{U}=\frac{Q}{Q+2 \pi \alpha_{g} \widetilde{\Pi}^{R}(\omega, \boldsymbol{q})}, \quad \widetilde{\Pi}^{R}(\omega, \boldsymbol{q})=\frac{v_{g}^{2}}{2 T} \Pi^{R}(\omega, \boldsymbol{q}) .
$$

For analytical estimates in the degenerate regime one can use the usual Thomas-Fermi static screening $[55,58]$

$$
\widetilde{U}=\frac{\widetilde{Q}}{\widetilde{Q}+2 \alpha_{g}} .
$$

In full units the inverse screening length is $\varkappa=N \alpha_{g} k_{F}$. The use of the static screening in Eq. (16) can be justified by the fact that in this integral the contribution of the region $Q \sim W$ is explicitly suppressed, while outside of this region for $W<Q<x$ the polarization operator in graphene is well approximated by a constant $[55,58]$.

Taking into account static screening, we find for the momentum integral $J_{33}$ in the opposite order of limits (first, $x \rightarrow \infty$, then $\alpha \rightarrow 0$ )

$$
\lim _{\alpha_{g} \rightarrow 0} \lim _{x \rightarrow \infty} J_{33} \approx \ln \frac{1}{2 \alpha_{g}}-\frac{7}{4}+\frac{W^{2}}{2 x^{2} \alpha_{g}^{2}}\left(1-\ln \frac{2 \alpha_{g} x}{W}\right) .
$$

The first two terms are identical for all integrals (B2f), so again we need to keep the subleading term. Combined with 
the rest of the integrals (B3) this leads to the result

$$
\eta(\mu \gg T)=\frac{3 \mu^{4}}{128 \pi^{2} \alpha_{g}^{2} v_{g}^{2} T^{2}} \frac{1}{\ln \frac{1}{\alpha_{g}}-\delta_{2}},
$$

where $\delta_{2}=7 / 4+\ln 2 \approx 2.44$. The factor $\delta_{2}$ is kept in the denominator in order to emphasize that the logarithmic dependence on the coupling constant is only valid in the limit $\alpha_{g} \rightarrow 0$ such that $\left|\ln \alpha_{g}\right| \gg 1$. For any practical value of the coupling constant Eq. (29) is negative and thus invalid. Instead of the limit $\alpha_{g} \rightarrow 0$, one has to consider the full expression for $J_{33}$.

The leading parametric dependence in Eq. (29) (up to the correction, $\delta_{2}$ ) was first suggested in Ref. [27]. The numerical prefactor that can be found in Ref. [40] appears to be twice as large as ours.

In gated structures screening is modified by the presence of the gate [56]. In particular, the "bare" Coulomb interaction $U_{0}$ should be replaced by

$$
U_{0} \rightarrow U_{0}^{g}=\frac{2 \pi e^{2}}{q}\left(1-e^{-2 q d}\right),
$$

where $d$ is the distance to the gate. This form assumes the single gate device. Note that the experiments of Ref. [4] were performed on double gate devices as well. In the latter case, the effective Coulomb interaction has a more complicated form. However, if the gate is placed far enough from the graphene sample (Ref. [4] reports the thickness of the insulating layer to be about $d=50 \mathrm{~nm}$ ), such that $d \gg 1 / \varkappa$, then the screening effect of the gate may be neglected.

\section{B. Kinematic viscosity in zero field}

Kinematic viscosity in graphene as a function of the carrier density is shown in Fig. 5, where we plot our results in physical units taking into account the renormalizations (20) and (27) as well as the dynamical screening (28). The results are in a reasonably good agreement with the experimental data reported in Fig. 4 of Ref. [4]: the theoretical values are of the same order of magnitude, $v \sim 0.1 \mathrm{~m}^{2} / \mathrm{s}$,

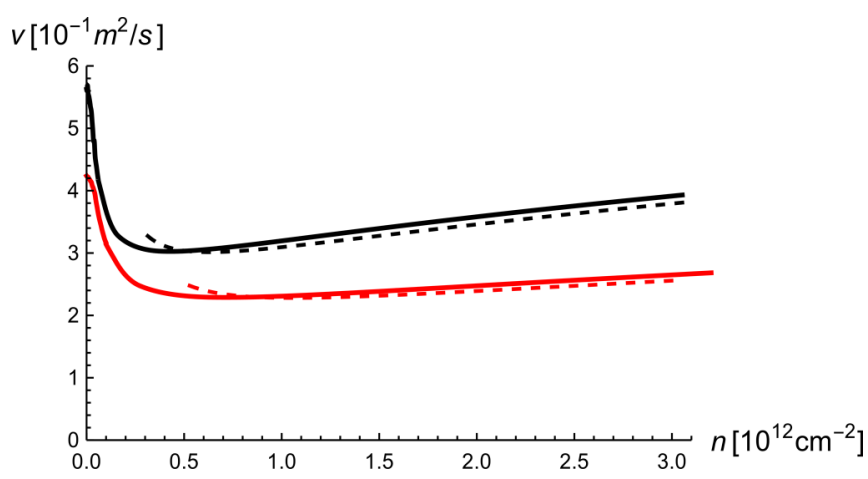

FIG. 5. Kinematic viscosity in graphene as a function of the carrier density. The solid lines are the result of the direct numerical evaluation of Eq. (27) with $\alpha_{g}=0.5$ and dynamical screening, Eq. (28). Dashed curves show the "Fermi-liquid" asymptotics, based on Eq. (16) with static screening. The lower dataset (shown in red) corresponds to $T=280 \mathrm{~K}$, the upper (black)—to $T=220 \mathrm{~K}$. The temperatures and the density range are taken from Fig. 4 of Ref. [4]. as the experimental ones, the density dependence in the range $n \approx 1 \div 3 \times 10^{12} \mathrm{~cm}^{-2}$ is rather weak, and the overall value decreases slightly with the temperature increase from $T=220 \mathrm{~K}$ to $T=280 \mathrm{~K}$.

Our results were obtained assuming the value $\alpha_{g} \approx 0.5$ for the "bare" coupling constant (as reported in the Supplemental Material to Ref. [4]). The "bare" velocity in graphene was taken as $v_{g}=10^{6} \mathrm{~m} / \mathrm{s}$. Then at $T=300 \mathrm{~K}$, the dimensionful prefactor in Eq. (27) has a value

$$
\left.\frac{v_{g}^{2}}{\alpha_{g}^{2} T} \rightarrow \frac{\hbar v_{g}^{2}}{\alpha_{g}^{2} k_{B} T}\right|_{T=300 \mathrm{~K}} \approx 0.1 \frac{\mathrm{m}^{2}}{\mathrm{~s}},
$$

which ultimately determines the order of magnitude of the resulting kinematic viscosity.

Combining this prefactor with the asymptotic behavior of $\tilde{\eta}$ [which can be read off Eqs. (17) or (29) disregarding the logarithm] and $\tilde{n}_{E} \approx x^{3}\left(1+\pi^{2} / x^{2}\right) / 6$ [from Eq. (A3)], we estimate the dominant temperature dependence of the kinematic viscosity (11) in the degenerate regime as

$$
v(\mu \gg T) \propto \frac{v_{g}^{2} \mu}{\alpha_{g}^{2} T^{2}} \frac{1}{1+\pi^{2} T^{2} / \mu^{2}} .
$$

This "naive" estimate neglects the temperature dependence arising from the renormalizations and the logarithmic factors in Eq. (17) [or Eq. (29)]. Nevertheless, the true temperature dependence is not far off as shown in Fig. 6 where we plot Eq. (31) together with the "Fermi-liquid" asymptotics for the kinematic viscosity (11) based on Eq. (16) and static screening (a reasonable approximation in the degenerate regime, see Fig. 5; the dashed curve is vertically shifted for clarity).

Similarly, we can estimate the temperature dependence of the kinematic viscosity at charge neutrality. Using Eq. (13) and the relation $n_{E}(\mu=0) \propto T^{3}$, we find

$$
\nu(\mu=0) \propto \frac{v_{g}^{2}}{\alpha_{g}^{2} T} \text {. }
$$

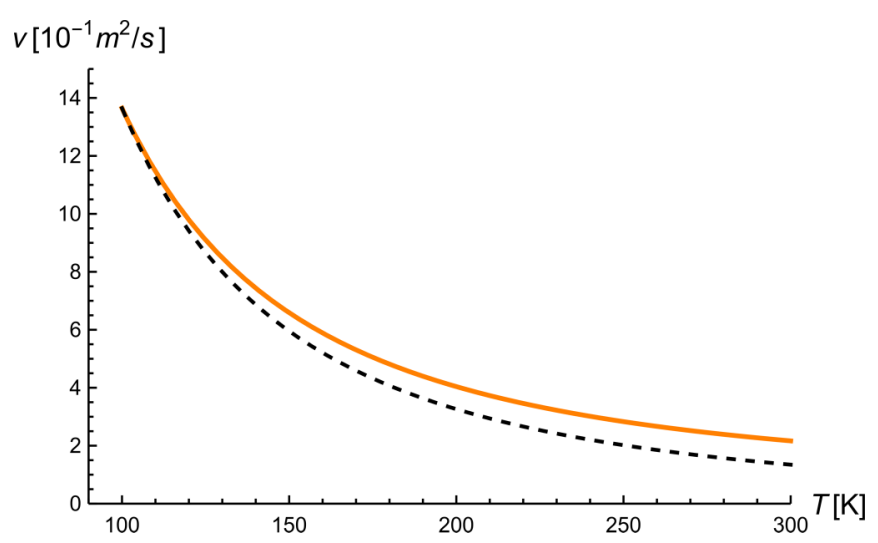

FIG. 6. Kinematic viscosity in graphene as a function of temperature evaluated at the carrier density $n=2 \times 10^{12} \mathrm{~cm}^{-2}$, where the "Fermi-liquid" asymptotics yields excellent agreement with the direct numerical evaluation of Eq. (27). The solid line shows the "Fermi-liquid" asymptotics for the kinematic viscosity (11) based on Eq. (16). The dashed line shows the "naive" temperature dependence (31), vertically shifted for clarity. The temperature and density ranges are taken from Fig. 3 of Ref. [14]. 
Again, the true temperature dependence will be slightly different due to the renormalization factors (the screening length at charge neutrality is determined by temperature and hence does not lead to any additional temperature dependence).

For the higher temperature range shown in Fig. 6 the kinematic viscosity may be fitted [27] by another power law, $v \propto T^{-1.5}$. However, this is an intermediate regime: for higher temperatures, $T \geqslant 350 \mathrm{~K}$, the calculated temperature dependence shows clear deviations from this behavior.

Finally, typical theoretical values of the kinematic viscosity shown in Fig. 5 differ from those reported in Ref. [4] by about a factor of 3. Our calculation does not involve any fitting parameters and does not take into account any particular features of the experimental device, e.g., screening by the gate and disorder scattering. As we have already mentioned, the renormalization group calculation leading to the factors of $R_{\Lambda}$ is approximate, so that we do not expect to find perfect agreement with the data. A true test of the theory would be to calculate the quantities actually measured in the experiment (e.g., the nonlocal resistivity [4], $R_{V}$ ) for realistic sample geometries. The results of such calculations will be reported in a subsequent publication.

\section{Hall viscosity}

Defining the "kinematic" counterpart of the Hall viscosity similarly to Eq. (11),

$$
v_{H}=\frac{v_{g}^{2} \eta_{H}}{W},
$$

and using the same RG approach, we find that $v_{H}$ renormalizes similarly to Eq. (27)

$$
v_{H}(T, \mu, \alpha)=\frac{2 \pi v_{g}^{2} \tilde{\eta}_{H}\left(x, \gamma_{B}\right)}{3 N \alpha_{g}^{2} T \tilde{n}_{E}(x)} R_{\Lambda}^{4} .
$$

Here $\tilde{\eta}_{H}\left(x, \gamma_{B}\right)$ is defined according to Eq. (23). For $T=300 \mathrm{~K}, B=1 \mathrm{~T}$ and neglecting renormalizations, the dimensionless quantity $\gamma_{B}$ (in SI units) is given by

$$
\gamma_{B}=\left.\frac{\hbar|e| v_{g}^{2} B}{\alpha_{g}^{2} k_{B}^{2} T^{2}}\right|_{T=300 \mathrm{~K} ; B=1 \mathrm{~T}} \approx 3.939 .
$$

The resulting values are shown in Figs. 7 and 8 in physical units. The former shows $v_{H}$ as a function of the external magnetic field for a fixed value of the charge density, $n \approx 2 \times 10^{12} \mathrm{~cm}^{-2}$. The results shown in Fig. 7 significantly exceed the experimental values shown in Fig. 3 of Ref. [14]. The origin of this discrepancy is in the high power of the renormalization factor in Eqs. (34) and (24). Indeed, combining Eqs. (18b), (34), and (24), we find

$$
v_{H}=R_{\Lambda}^{4} \frac{\pi}{6} \frac{v_{g}^{2}}{\alpha_{g}^{2} T} \frac{\tilde{\eta}(x, \varkappa)}{\tilde{n}_{E}(x)} \frac{B B_{0}}{B^{2}+B_{0}^{2}},
$$

where the "reference field" $B_{0}$ introduced in Refs. $[14,52]$ is given by

$$
B_{0}=R_{\Lambda}^{-4} \frac{c \alpha_{g}^{2} T^{2}}{|e| v_{g}^{2} f(x, \varkappa)} .
$$

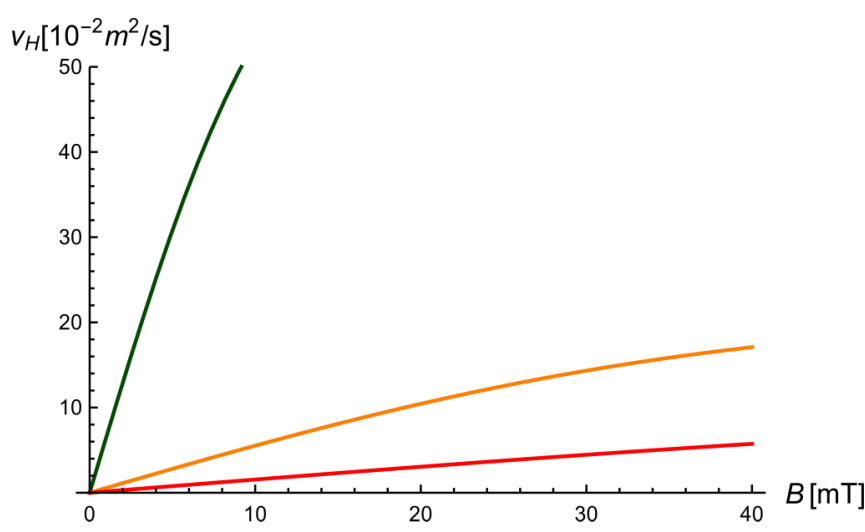

FIG. 7. Hall viscosity in graphene as a function of magnetic field. Solid lines show the "Fermi-liquid" asymptotics for the kinematic Hall viscosity (33) based on Eq. (16) with $\alpha_{g}=0.5$ for $n=2 \times 10^{12} \mathrm{~cm}^{-2}$ and three different temperatures, $T=100 \mathrm{~K}$, $T=220 \mathrm{~K}$, and $T=300 \mathrm{~K}$ (shown in green, orange, and red, respectively). The density, temperature, and field range are taken from Fig. 3 of Ref. [14].

Here the dimensionless function $f(x, \varkappa)$ is defined similarly to Eq. (24) with the dominant contribution coming from the scattering time $\tilde{\tau}_{11}$ [defined in Eq. (B3)].

The temperature dependence of $v_{H}$ shown in Fig. 8 for weak magnetic fields, $B \ll B_{0}$, and in the degenerate regime can be extracted from Eqs. (35) and (B3),

$$
v_{H}\left(\mu \gg T ; B \ll B_{0}\right) \propto \frac{|e| v_{g}^{4}}{c \alpha_{g}^{4} T^{3}} \frac{1}{1+\pi^{2} T^{2} / \mu^{2}} .
$$

Similarly to Eqs. (31) and (32) this expression disregards additional temperature dependence from the renormalizations and screening [i.e., the logarithmic factors in Eqs. (17) or Eq. (29)]. Nevertheless, it accounts for the temperature

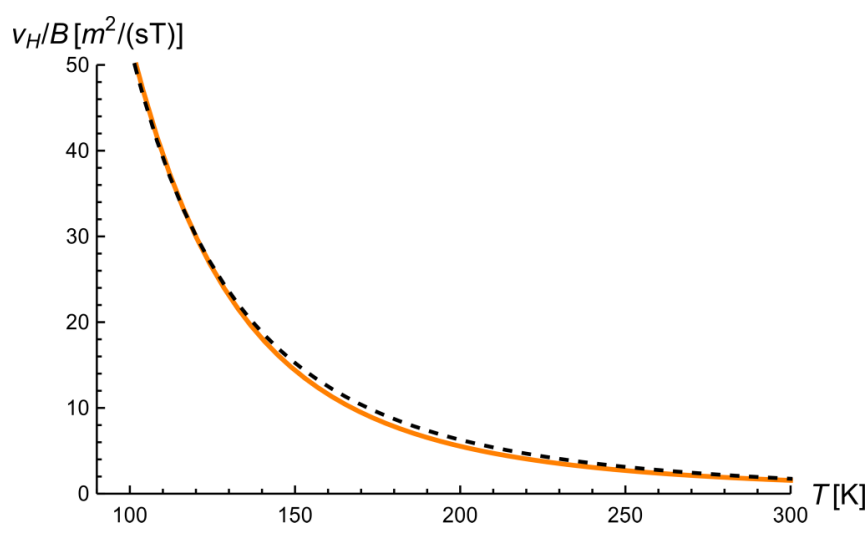

FIG. 8. Temperature dependence of the kinematic Hall viscosity. The solid line shows the "Fermi-liquid" asymptotics for the kinematic Hall viscosity (33) based on Eq. (16) with $\alpha_{g}=0.5$ for $n=2 \times 10^{12} \mathrm{~cm}^{-2}$. The dashed line shows the "naive" temperature dependence (36), vertically shifted for clarity. The density and temperature ranges are taken from Fig. 3 of Ref. [14]. 
dependence of the Hall viscosity in the degenerate regime rather well, see Fig. 8.

\section{SUMMARY}

In conclusion, we have calculated the shear and Hall viscosities in graphene at arbitrary doping levels and classical (nonquantizing) magnetic fields using the kinetic theory approach combined with the RG analysis. The shear viscosity in graphene exhibits a monotonous growth as a function of carrier density (or chemical potential) from a small value at charge neutrality, see Eqs. (17) and (29). In contrast, the kinematic viscosity (11) remains of the same order of magnitude at all doping levels, see Fig. 5: $v_{H}(n)$ decays from the initial value at $n=0$ and then passes through a minimum followed by a (initially weak) growth in the degenerate regime. This behavior follows from the nontrivial density dependence of the enthalpy (or equivalently, energy density). The appearance of the enthalpy in the definition of kinematic viscosity (11) is a characteristic feature of the electronic system in graphene (in contrast, the usual definition of the kinematic viscosity involves the mass density [41]).

The field dependence of the shear and Hall viscosities shown in Figs. 2 and 3 can be expected on general grounds. In particular, the Hall viscosity vanishes at zero magnetic field as well as for classically strong fields and hence has to exhibit a maximum. In the degenerate regime, both viscosities are well described by the semiclassical expressions (18) first suggested in Ref. [48]. For smaller densities the shape of the field dependence of $\eta$ and $\eta_{H}$ deviates from Eqs. (18) but remains very similar. The only exception to this argument is the field dependence of the shear viscosity at charge neutrality, see Fig. 1, which remains finite in classically strong fields. This effect can be traced to the complete decoupling of the charge and energy currents at charge neutrality.

In the degenerate regime of large charge densities, our results are in a reasonably good agreement with the available experimental evidence. The quantitative discrepancies between the theoretical values shown in Figs. 5-8 and the results of Refs. $[4,14]$ can be attributed to our use of the renormalization group resulting in relatively strong enhancement of the kinematic viscosity (11) and especially the kinematic Hall viscosity (33). Moreover, our calculation does not include sample-specific details such as screening by the gate and disorder scattering. The latter can be expected to reduce the viscosity values. In any case, a true test of the theory would be a calculation of several distinct quantities actually measured in the experiment for realistic sample geometries (using, e.g., the experimentally measured [66-68] values for renormalized velocity). Such results will be reported elsewhere.

\section{ACKNOWLEDGMENTS}

We thank I. V. Gornyi, A. D. Mirlin, and J. Schmalian for fruitful discussions. This work was supported by the German Research Foundation DFG within FLAG-ERA Joint Transnational Call (Project GRANSPORT) and the MEPhI Academic Excellence Project, Contract No. 02.a03.21.0005 (BNN) and the European Union's Horizon 2020 research and innovation programme under the Marie Sklodowska-Curie Grant Agreement No. 701647 (MS).

\section{APPENDIX A: LOCAL EQUILIBRIUM QUANTITIES}

Under the assumption of local equilibrium, the macroscopic quantities appearing in the hydrodynamic equations are given by

$$
\begin{gathered}
n=n_{+}-n_{-}=\frac{T^{2}}{v_{g}^{2}} \frac{g_{2}\left(\mu_{+} / T\right)-g_{2}\left(-\mu_{-} / T\right)}{\left(1-u^{2} / v_{g}^{2}\right)^{3 / 2}} \\
n_{I}=n_{+, 0}+n_{-}=\frac{T^{2}}{v_{g}^{2}} \frac{g_{2}\left(\mu_{+} / T\right)+g_{2}\left(-\mu_{-} / T\right)}{\left(1-u^{2} / v_{g}^{2}\right)^{3 / 2}}, \\
g_{2}\left(\frac{\mu}{T}\right)=-\frac{N}{2 \pi} \operatorname{Li}_{2}\left(-e^{\mu / T}\right)
\end{gathered}
$$

where $\mathrm{Li}_{n}$ is the polylogarithm,

$$
\begin{aligned}
n_{E}=2 \frac{T^{3}}{v_{g}^{2}} \frac{1+u^{2} /\left(2 v_{g}^{2}\right)}{\left(1-u^{2} / v_{g}^{2}\right)^{5 / 2}}\left[g_{3}\left(\frac{\mu_{+}}{T}\right)+g_{3}\left(-\frac{\mu_{-}}{T}\right)\right] \\
g_{3}\left(\frac{\mu}{T}\right)=-\frac{N}{2 \pi} \operatorname{Li}_{3}\left(-e^{\mu / T}\right) \\
P=n_{E} \frac{1-u^{2} / v_{g}^{2}}{2+u^{2} / v_{g}^{2}} \\
W=n_{E}+P=\frac{3 n_{E}}{2+u^{2} / v_{g}^{2}} \\
\boldsymbol{j}=n \boldsymbol{u}, \quad \boldsymbol{j}_{I}=n_{I} \boldsymbol{u} \\
\boldsymbol{j}_{E}=v_{g}^{2} \boldsymbol{n}_{\boldsymbol{k}}=W \boldsymbol{u} \\
\Pi_{E}^{\alpha \beta}=P \delta_{\alpha \beta}+v_{g}^{-2} W u_{\alpha} u_{\beta}
\end{aligned}
$$

\section{APPENDIX B: GENERAL EXPRESSIONS FOR THE VISCOSITY COEFFICIENTS}

The general expressions for the shear and Hall viscosities in graphene

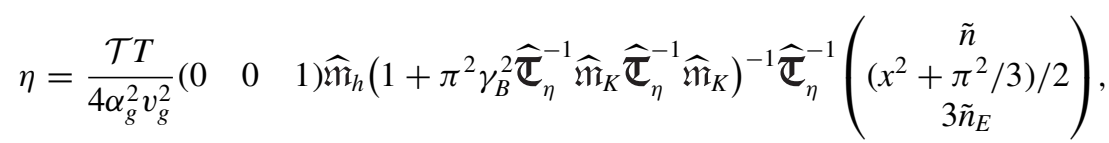

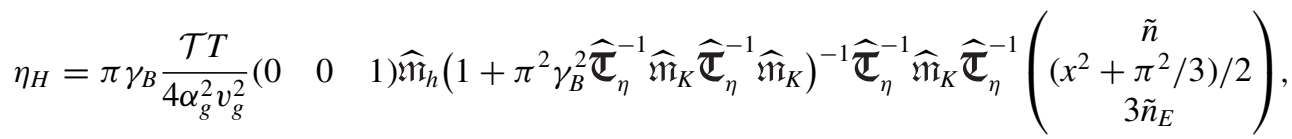


with the following notations

$$
\begin{aligned}
\gamma_{B} & =\frac{|e| v_{g}^{2} B}{\alpha_{g}^{2} c T^{2}}, \\
\mathcal{T} & =T\left[\ln \left(1+e^{\mu_{+} / T}\right)+\ln \left(1+e^{-\mu_{-} / T}\right)\right] \rightarrow \mathcal{T}=2 T \ln 2 \cosh \frac{\mu}{2 T},
\end{aligned}
$$

where the last expression is obtained in the limit $\mu_{ \pm}=\mu$, that is used hereafter,

$$
\begin{aligned}
& \tilde{n}(x)=-\mathrm{Li}_{2}\left(-e^{x}\right)+\mathrm{Li}_{2}\left(-e^{-x}\right), \quad \tilde{n}_{E}(x)=-\mathrm{Li}_{3}\left(-e^{x}\right)-\mathrm{Li}_{3}\left(-e^{-x}\right), \quad x=\frac{\mu}{T}, \\
& \widehat{\mathfrak{w}}_{h}=\left(\begin{array}{ccc}
1 & \frac{x T}{\mathcal{T}} & 2 \tilde{n}_{\overline{\mathcal{T}}}^{\frac{T}{2}} \\
\frac{x T}{\mathcal{T}} & 1 & {\left[x^{2}+\frac{\pi^{2}}{3}\right] \frac{T}{\mathcal{T}}} \\
2 \tilde{n} \frac{T}{\mathcal{T}} & {\left[x^{2}+\frac{\pi^{2}}{3}\right] \frac{T}{\mathcal{T}}} & 6 \tilde{n}_{E} \frac{T}{\mathcal{T}}
\end{array}\right), \\
& \widehat{\mathfrak{m}}_{K}=\left(\begin{array}{ccc}
\tanh \frac{x}{2} & 1 & \frac{\mathcal{T}}{T} \\
1 & \tanh \frac{x}{2} & x \\
\frac{\mathcal{T}}{T} & x & 2 \tilde{n}
\end{array}\right) \\
& \widehat{\mathbb{U}}_{\eta}=\frac{2 \pi}{\alpha_{g}^{2}} \frac{\mathcal{T}}{N T^{2}}\left(\begin{array}{ccc}
\tilde{\tau}_{11}^{-1} & \tilde{\tau}_{12}^{-1} & \tilde{\tau}_{13}^{-1} \\
\tilde{\tau}_{12}^{-1} & \tilde{\tau}_{22}^{-1} & \tilde{\tau}_{23}^{-1} \\
\tilde{\tau}_{13}^{-1} & \tilde{\tau}_{23}^{-1} & \tilde{\tau}_{33}^{-1}
\end{array}\right) \text {. }
\end{aligned}
$$

The "scattering rates" $\tilde{\tau}_{i j}^{-1}$ are obtained from the collision integral within the three-mode approximation [25] and are given by

$$
\frac{1}{\tilde{\tau}_{i j}}=(2 \pi)^{2} \alpha_{g}^{2} N T\left[\frac{N T}{v_{g}^{2} \partial n_{0} / \partial \mu}\right] \int \frac{d^{2} Q}{(2 \pi)^{2}} \frac{d W}{2 \pi} \frac{|\widetilde{U}|^{2}}{\sinh ^{2} W}\left[Y_{00} \widetilde{Y}_{i j}-\widetilde{Y}_{0 j} \widetilde{Y}_{0 i}\right],
$$

where

$$
\begin{aligned}
& Y_{00}(\omega, \boldsymbol{q})=\frac{1}{4 \pi}\left[\frac{\theta(|\Omega| \leqslant 1)}{\sqrt{1-\Omega^{2}}} \mathcal{Z}_{0}^{>}\left[I_{1}\right]+\frac{\theta(|\Omega| \geqslant 1)}{\sqrt{\Omega^{2}-1}} \mathcal{Z}_{0}^{<}\left[I_{1}\right]\right] \\
& \widetilde{Y}_{01}(\omega, \boldsymbol{q})=-\frac{1}{\pi}\left[\theta(|\Omega| \leqslant 1) \Omega \sqrt{1-\Omega^{2}} \mathcal{Z}_{5}^{>}[I]+\theta(|\Omega| \geqslant 1) \Omega \sqrt{\Omega^{2}-1} \mathcal{Z}_{5}^{<}[I]\right] \\
& \widetilde{Y}_{02}(\omega, \boldsymbol{q})=\frac{1}{\pi}\left[\theta(|\Omega| \leqslant 1) \Omega \sqrt{1-\Omega^{2}} \mathcal{Z}_{5}^{>}\left[I_{1}\right]-\frac{1}{2} \theta(|\Omega| \geqslant 1) \operatorname{sgn}(\Omega) \sqrt{\Omega^{2}-1} \widetilde{\mathcal{Z}}_{4}^{<}\left[I_{1}\right]\right]+\frac{1}{4 \pi} \theta(|\Omega| \geqslant 1) \frac{\operatorname{sgn}(\Omega)}{\sqrt{\Omega^{2}-1}} \mathcal{Z}_{0}^{<}\left[I_{1}\right] \\
& \tilde{Y}_{03}(\omega, \boldsymbol{q})=Q \Omega Y_{00}(\omega, \boldsymbol{q})+\frac{1}{2 \pi} Q \Omega\left[\theta(|\Omega| \leqslant 1) \sqrt{1-\Omega^{2}} \mathcal{Z}_{3}^{>}\left[I_{1}\right]-\theta(|\Omega| \geqslant 1) \sqrt{\Omega^{2}-1} \mathcal{Z}_{3}^{<}\left[I_{1}\right]\right] \\
& \widetilde{Y}_{11}(\omega, \boldsymbol{q})=\frac{1}{\pi}\left[\theta(|\Omega| \leqslant 1) \sqrt{1-\Omega^{2}} \mathcal{Z}_{4}^{>}\left[I_{1}\right]+\theta(|\Omega| \geqslant 1) \sqrt{\Omega^{2}-1} \mathcal{Z}_{4}^{<}\left[I_{1}\right]\right] \\
& \widetilde{Y}_{12}(\omega, \boldsymbol{q})=-\frac{1}{\pi} \theta(|\Omega| \leqslant 1) \sqrt{1-\Omega^{2}} \mathcal{Z}_{4}^{>}[I], \\
& \widetilde{Y}_{13}(\omega, \boldsymbol{q})=-\frac{Q}{\pi}\left[\theta(|\Omega| \leqslant 1) \sqrt{1-\Omega^{2}} \mathcal{Z}_{5}^{>}[I]+\theta(|\Omega| \geqslant 1) \sqrt{\Omega^{2}-1} \mathcal{Z}_{5}^{<}[I]\right] \\
& \widetilde{Y}_{22}(\omega, \boldsymbol{q})=\frac{1}{\pi}\left[\theta(|\Omega| \leqslant 1) \sqrt{1-\Omega^{2}} \mathcal{Z}_{4}^{>}\left[I_{1}\right]-\theta(|\Omega| \geqslant 1) \sqrt{\Omega^{2}-1} \mathcal{Z}_{4}^{<}\left[I_{1}\right]+\frac{1}{4} \frac{\theta(|\Omega| \geqslant 1)}{\sqrt{\Omega^{2}-1}} \mathcal{Z}_{0}^{<}\left[I_{1}\right]\right] \\
& \widetilde{Y}_{23}(\omega, \boldsymbol{q})=\frac{Q}{\pi}\left[\theta(|\Omega| \leqslant 1) \sqrt{1-\Omega^{2}} \mathcal{Z}_{5}^{>}\left[I_{1}\right]-\theta(|\Omega| \geqslant 1)|\Omega| \sqrt{\Omega^{2}-1} \mathcal{Z}_{4}^{<}\left[I_{1}\right]+\frac{|\Omega|}{4} \frac{\theta(|\Omega| \geqslant 1)}{\sqrt{\Omega^{2}-1}} \mathcal{Z}_{0}^{<}\left[I_{1}\right]\right] \\
& \widetilde{Y}_{33}(\omega, \boldsymbol{q})=\frac{Q^{2}}{\pi}\left[\theta(|\Omega| \leqslant 1) \sqrt{1-\Omega^{2}} \mathcal{Z}_{3}^{>}\left[I_{1}\right]-\theta(|\Omega| \geqslant 1) \sqrt{\Omega^{2}-1} \mathcal{Z}_{3}^{<}\left[I_{1}\right]\right]+Q^{2} \Omega^{2} Y_{00}(\omega, \boldsymbol{q}) \\
&
\end{aligned}
$$

These functions are expressed in terms of the integrals

$$
\mathcal{Z}_{0}^{>}[I]=\int_{1}^{\infty} d z \sqrt{z^{2}-1} I(z), \quad \mathcal{Z}_{0}^{<}[I]=\int_{0}^{1} d z \sqrt{1-z^{2}} I(z),
$$




$$
\begin{array}{ll}
\mathcal{Z}_{3}^{>}[I]=\int_{1}^{\infty} d z \frac{\left(z^{2}-1\right)^{3 / 2}}{z^{2}-\Omega^{2}} I(z), & \mathcal{Z}_{3}^{<}[I]=\int_{0}^{1} d z \frac{\left(1-z^{2}\right)^{3 / 2}}{\Omega^{2}-z^{2}} I(z), \\
\mathcal{Z}_{4}^{>}[I]=\int_{1}^{\infty} d z \frac{\left(z^{2}-1\right)^{3 / 2}}{\left(z^{2}-\Omega^{2}\right)^{2}} I(z), & \mathcal{Z}_{4}^{<}[I]=\int_{0}^{1} d z \frac{\left(1-z^{2}\right)^{3 / 2}}{\left(\Omega^{2}-z^{2}\right)^{2}} I(z), \quad \widetilde{\mathcal{Z}}_{4}^{<}[I]=\int_{0}^{1} d z \frac{\left(1-z^{2}\right)^{3 / 2}}{\left(\Omega^{2}-z^{2}\right)^{2}}\left(\Omega^{2}+z^{2}\right) I(z), \\
\mathcal{Z}_{5}^{>}[I]=\int_{1}^{\infty} d z \frac{z\left(z^{2}-1\right)^{3 / 2}}{\left(z^{2}-\Omega^{2}\right)^{2}} I(z), & \mathcal{Z}_{5}^{<}[I]=\int_{0}^{1} d z \frac{z\left(1-z^{2}\right)^{3 / 2}}{\left(\Omega^{2}-z^{2}\right)^{2}} I(z),
\end{array}
$$

that are evaluated for either of the two functions

$$
\begin{gathered}
I_{1}(z)=\tanh \frac{z Q+W+x}{2}+\tanh \frac{z Q+W-x}{2}-\tanh \frac{z Q-W+x}{2}-\tanh \frac{z Q-W-x}{2}, \\
I(z)=\tanh \frac{z Q+W+x}{2}-\tanh \frac{z Q+W-x}{2}-\tanh \frac{z Q-W+x}{2}+\tanh \frac{z Q-W-x}{2} .
\end{gathered}
$$

The frequency and momentum are expressed in terms of the dimensionless variables

$$
Q=\frac{v_{g} \boldsymbol{q}}{2 T}, \quad W=\frac{\omega}{2 T} .
$$

Finally, the Coulomb interaction has the form

$$
U(\omega, \boldsymbol{q})=\frac{2 \pi e^{2}}{q} \widetilde{U}=\frac{2 \pi \alpha_{g} v_{g}}{q} \widetilde{U}, \quad \alpha_{g}=\frac{e^{2}}{v_{g} \varepsilon},
$$

where $\varepsilon$ is the dielectric constant of the dielectric environment and the dimensionless factor $\widetilde{U}$ accounts for screening.

[1] J. G. Checkelsky and N. P. Ong, Phys. Rev. B 80, 081413(R) (2009).

[2] Y. M. Zuev, W. Chang, and P. Kim, Phys. Rev. Lett. 102, 096807 (2009).

[3] D. A. Abanin, S. V. Morozov, L. A. Ponomarenko, R. V. Gorbachev, A. S. Mayorov, M. I. Katsnelson, K. Watanabe, T. Taniguchi, K. S. Novoselov, L. S. Levitov et al., Science 332, 328 (2011).

[4] D. A. Bandurin, I. Torre, R. Krishna Kumar, M. Ben Shalom, A. Tomadin, A. Principi, G. H. Auton, E. Khestanova, K. S. Novoselov, I. V. Grigorieva et al., Science 351, 1055 (2016).

[5] J. Crossno, J. K. Shi, K. Wang, X. Liu, A. Harzheim, A. Lucas, S. Sachdev, P. Kim, T. Taniguchi, K. Watanabe et al., Science 351, 1058 (2016).

[6] P. J. Moll, P. Kushwaha, N. Nandi, B. Schmidt, and A. P. Mackenzie, Science 351, 1061 (2016).

[7] F. Ghahari, H.-Y. Xie, T. Taniguchi, K. Watanabe, M. S. Foster, and P. Kim, Phys. Rev. Lett. 116, 136802 (2016).

[8] R. Krishna Kumar, D. A. Bandurin, F. M. D. Pellegrino, Y. Cao, A. Principi, H. Guo, G. H. Auton, M. Ben Shalom, L. A. Ponomarenko, G. Falkovich et al., Nat. Phys. 13, 1182 (2017).

[9] D. A. Bandurin, A. V. Shytov, L. S. Levitov, R. K. Kumar, A. I. Berdyugin, M. Ben Shalom, I. V. Grigorieva, A. K. Geim, and G. Falkovich, Nat. Commun. 9, 4533 (2018).

[10] B. A. Braem, F. M. D. Pellegrino, A. Principi, M. Röösli, C. Gold, S. Hennel, J. V. Koski, M. Berl, W. Dietsche, W. Wegscheider et al., Phys. Rev. B 98, 241304(R) (2018).

[11] A. Jaoui, B. Fauqué, C. W. Rischau, A. Subedi, C. Fu, J. Gooth, N. Kumar, V. Süß, D. L. Maslov, C. Felser et al., npj Quantum Materials 3, 64 (2018).

[12] L. Ella, A. Rozen, J. Birkbeck, M. Ben-Shalom, D. Perello, J. Zultak, T. Taniguchi, K. Watanabe, A. K. Geim, S. Ilani et al., Nat. Nanotechnol. 14, 480 (2019).
[13] P. Gallagher, C.-S. Yang, T. Lyu, F. Tian, R. Kou, H. Zhang, K. Watanabe, T. Taniguchi, and F. Wang, Science 364, 158 (2019).

[14] A. I. Berdyugin, S. G. Xu, F. M. D. Pellegrino, R. K. Kumar, A. Principi, I. Torre, M. B. Shalom, T. Taniguchi, K. Watanabe, I. V. Grigorieva et al., Science 364, 162 (2019).

[15] S. A. Hartnoll, P. K. Kovtun, M. Müller, and S. Sachdev, Phys. Rev. B 76, 144502 (2007).

[16] A. B. Kashuba, Phys. Rev. B 78, 085415 (2008).

[17] M. Müller, L. Fritz, and S. Sachdev, Phys. Rev. B 78, 115406 (2008).

[18] L. Fritz, J. Schmalian, M. Müller, and S. Sachdev, Phys. Rev. B 78, 085416 (2008).

[19] M. Müller and S. Sachdev, Phys. Rev. B 78, 115419 (2008).

[20] M. J. Bhaseen, A. G. Green, and S. L. Sondhi, Phys. Rev. B 79, 094502 (2009).

[21] M. S. Foster and I. L. Aleiner, Phys. Rev. B 79, 085415 (2009).

[22] M. Müller, J. Schmalian, and L. Fritz, Phys. Rev. Lett. 103, 025301 (2009).

[23] I. Torre, A. Tomadin, A. K. Geim, and M. Polini, Phys. Rev. B 92, 165433 (2015).

[24] B. N. Narozhny, I. V. Gornyi, M. Titov, M. Schütt, and A. D. Mirlin, Phys. Rev. B 91, 035414 (2015).

[25] U. Briskot, M. Schütt, I. V. Gornyi, M. Titov, B. N. Narozhny, and A. D. Mirlin, Phys. Rev. B 92, 115426 (2015).

[26] L. Levitov and G. Falkovich, Nat. Phys. 12, 672 (2016).

[27] A. Principi, G. Vignale, M. Carrega, and M. Polini, Phys. Rev. B 93, 125410 (2016).

[28] A. Lucas, J. Crossno, K. C. Fong, P. Kim, and S. Sachdev, Phys. Rev. B 93, 075426 (2016).

[29] G. Falkovich and L. Levitov, Phys. Rev. Lett. 119, 066601 (2017).

[30] H. Guo, E. Ilseven, G. Falkovich, and L. S. Levitov, Proc. Natl. Acad. Sci. USA 114, 3068 (2017). 
[31] Y. Seo, G. Song, P. Kim, S. Sachdev, and S.-J. Sin, Phys. Rev. Lett. 118, 036601 (2017).

[32] P. J. Ledwith, H. Guo, A. V. Shytov, and L. Levitov, arXiv: 1708.02376.

[33] A. Shytov, J. F. Kong, G. Falkovich, and L. Levitov, Phys. Rev. Lett. 121, 176805 (2018).

[34] D. Y. H. Ho, I. Yudhistira, N. Chakraborty, and S. Adam, Phys. Rev. B 97, 121404(R) (2018).

[35] J. M. Link, D. E. Sheehy, B. N. Narozhny, and J. Schmalian, Phys. Rev. B 98, 195103 (2018).

[36] E. I. Kiselev and J. Schmalian, Phys. Rev. B 99, 035430 (2019).

[37] H.-Y. Xie and A. Levchenko, Phys. Rev. B 99, 045434 (2019).

[38] I. S. Burmistrov, M. Goldstein, M. Kot, V. D. Kurilovich, and P. D. Kurilovich, Phys. Rev. Lett. 123, 026804 (2019).

[39] B. N. Narozhny, I. V. Gornyi, A. D. Mirlin, and J. Schmalian, Ann. Phys. 529, 1700043 (2017).

[40] A. Lucas and K. C. Fong, J. Phys.: Condens. Matter 30, 053001 (2018).

[41] L. D. Landau and E. M. Lifshitz, Fluid Mechanics (Pergamon Press, London, 1959).

[42] B. Bradlyn, M. Goldstein, and N. Read, Phys. Rev. B 86, 245309 (2012).

[43] I. M. Khalatnikov, Zh. Eksp. Teor. Fiz. 29, 253 (1956) [Sov. Phys. JETP-USSR 2, 169 (1956)].

[44] E. M. Lifshitz and L. P. Pitaevskii, Physical Kinetics (Pergamon Press, London, 1981).

[45] A. A. Abrikosov and I. M. Khalatnikov, Rep. Prog. Phys. 22, 329 (1959).

[46] J. M. Link, B. N. Narozhny, E. I. Kiselev, and J. Schmalian, Phys. Rev. Lett. 120, 196801 (2018).

[47] D. E. Sheehy and J. Schmalian, Phys. Rev. Lett. 99, 226803 (2007).

[48] P. S. Alekseev, Phys. Rev. Lett. 117, 166601 (2016).

[49] T. Scaffidi, N. Nandi, B. Schmidt, A. P. Mackenzie, and J. E. Moore, Phys. Rev. Lett. 118, 226601 (2017).

[50] M. S. Steinberg, Phys. Rev. 109, 1486 (1958).
[51] A. Tomadin, G. Vignale, and M. Polini, Phys. Rev. Lett. 113, 235901 (2014).

[52] F. M. D. Pellegrino, I. Torre, and M. Polini, Phys. Rev. B 96, 195401 (2017).

[53] B. N. Narozhny, arXiv:1905.09686.

[54] M. I. Katsnelson, Graphene (Cambridge University Press, Cambridge, 2012).

[55] M. Schütt, P. M. Ostrovsky, I. V. Gornyi, and A. D. Mirlin, Phys. Rev. B 83, 155441 (2011).

[56] I. L. Aleiner and B. I. Shklovskii, Phys. Rev. B 49, 13721 (1994).

[57] P. S. Alekseev, A. P. Dmitriev, I. V. Gornyi, V. Y. Kachorovskii, B. N. Narozhny, M. Schütt, and M. Titov, Phys. Rev. Lett. 114, 156601 (2015).

[58] B. N. Narozhny, M. Titov, I. V. Gornyi, and P. M. Ostrovsky, Phys. Rev. B 85, 195421 (2012).

[59] J. González, F. Guinea, and M. A. H. Vozmediano, Phys. Rev. B 59, R2474 (1999).

[60] J. González, F. Guinea, and M. Vozmediano, Nucl. Phys. B 424, 595 (1994).

[61] A. J. Millis, Phys. Rev. B 48, 7183 (1993).

[62] N. M. R. Peres, F. Guinea, and A. H. Castro Neto, Phys. Rev. B 73, 125411 (2006).

[63] A. A. Kozikov, A. K. Savchenko, B. N. Narozhny, and A. V. Shytov, Phys. Rev. B 82, 075424 (2010).

[64] M. Titov, R. V. Gorbachev, B. N. Narozhny, T. Tudorovskiy, M. Schütt, P. M. Ostrovsky, I. V. Gornyi, A. D. Mirlin, M. I. Katsnelson, K. S. Novoselov et al., Phys. Rev. Lett. 111, 166601 (2013).

[65] E. H. Hwang, B. Y.-K. Hu, and S. Das Sarma, Phys. Rev. Lett. 99, 226801 (2007).

[66] D. A. Siegel, C.-H. Park, C. Hwang, J. Deslippe, A. V. Fedorov, S. G. Louie, and A. Lanzara, PNAS 108, 11365 (2011).

[67] D. C. Elias, R. V. Gorbachev, A. S. Mayorov, S. V. Morozov, A. A. Zhukov, P. Blake, L. A. Ponomarenko, I. V. Grigorieva, K. S. Novoselov, F. Guinea et al., Nat. Phys. 7, 701 (2011).

[68] C. Hwang, D. A. Siegel, S.-K. Mo, W. Regan, A. Ismach, Y. Zhang, A. Zettl, and A. Lanzara, Sci. Rep. 2, 590 (2012). 